\title{
UTILIDADE DA CLASSIFICAÇÃO INTERNACIONAL DA FUN- CIONALIDADE, INCAPACIDADE E SAÚDE: VERSÃO PARA CRIANÇAS E JOVENS (CIF-CJ) NO CONTEXTO DA INTER- VENÇÃO PRECOCE E DA EDUCAÇÃO ESPECIAL
}

\author{
Helena Rosário ${ }^{1}$ \\ Teresa Leal ${ }^{2}$ \\ Ana Isabel Pinto ${ }^{3}$ \\ Rune J. Simeonsson ${ }^{4}$
}

\begin{abstract}
Resumo: O presente artigo tem como objectivo reflectir acerca da utilidade da Classificação Internacional da Funcionalidade, Incapacidade e Saúde - Versão para Crianças e Jovens - CIF-CJ (WHO, 2007), no âmbito da Intervenção Precoce e da Educação Especial. É discutida a importância da CIF-CJ como operacionalização de um modelo teórico unificador e abrangente que, ao descrever as características da funcionalidade individual, considerando a participação da pessoa com base em processos interactivos pessoa-ambiente, ultrapassa as limitações da abordagem diagnóstica ao desenvolvimento. São apresentadas as linhas gerais de vários estudos realizados na Faculdade de Psicologia e de Ciências da Educação da Universidade do Porto, que têm como objectivo analisar a utilidade da classificação no processo de avaliação - intervenção na Educação Especial e na Intervenção Precoce.
\end{abstract}

Palavras-chave: funcionalidade, classificação, intervenção precoce, educação especial

The utility of the International Classification of Functioning, Disability and Health: Versions for children and youth (ICF-CY) in early intervention and special education contexts (Abstract): This paper discusses the utility of the International Classification of Functioning, Disability and Health - Children and Youth - ICF-CY (WHO, 2007) in the scope of Early Intervention and Early Childhood Special Education. We will discuss the relevance of the ICF-CY as an operationalization of an

\footnotetext{
${ }^{1}$ Faculdade de Psicologia e de Ciências da Educação, Universidade do Porto, Bolseira de Doutoramento da Fundação da Ciência e Tecnologia (SFRH/BD/27930/2006)

2 Faculdade de Psicologia e de Ciências da Educação, Universidade do Porto

${ }^{3}$ Faculdade de Psicologia e de Ciências da Educação, Universidade do Porto

${ }^{4}$ University of North Carolina at Chapel Hill, USA 
unifying and comprehensive theoretical approach. This approach describes the person's functioning while taking into account individual participation, based on an interactive person-environment process, thus overcoming shortcomings of the diagnostic approaches to development. The general lines of research that are being developed at the Faculdade de Psicologia e de Ciências da Educação da Universidade do Porto, concerning the utility of the classification in the assessment-intervention process in Early Intervention and Early Childhood Special Education will be presented.

Key-words: functioning, classification, early intervention, early childhood special education

A maioria dos estudos sobre o desenvolvimento humano reconhece já a sua natureza interactiva e salienta os contributos dos factores relativos à criança e ao meio na sua determinação (Pinto, Pessanha, Barros, Grande, Aguiar, C. \& Nunes, 2009). Neste sentido, considera-se que o desenvolvimento humano é complexo e multideterminado, sendo a interacção um conceito fulcral que é conceptualizado como a unidade de análise que descreve as trocas activas de um indivíduo com os acontecimentos físicos e sociais do seu ambiente (Bairrão, 2003). Dentro desta perspectiva, a incapacidade é vista como um conjunto complexo de condições que resultam da interacção pessoa-meio, constituindo um conceito relativo e dinâmico. É, pois, fundamental considerar que características, actividades e ambientes de crianças e jovens requerem formas próprias de avaliação e documentação.

Assim, a avaliação abrangente da funcionalidade é essencial para a elaboração de intervenções que sirvam de base à redução das limitações funcionais e ao aumento do bem-estar das crianças. A avaliação, no seu sentido mais lato, deve, assim, documentar as manifestações específicas da incapacidade na criança, podendo também contribuir para a sua descrição e para a avaliação da eficácia dos programas de intervenção (Berg \& Kleinman, 1998, como citado em Simeonsson, Pereira, \& Scarborough, 2003).

Esta conceptualização apresenta-se consistente com uma mudança do paradigma médico da deficiência para o chamado paradigma biopsicossocial, que passa a enquadrar as questões relativas às incapacidades e ao processo de avaliação-intervenção numa perspectiva onde o ambiente desempenha um papel primordial, ao influenciar a funcionalidade humana e a participação dos indivíduos nos seus contextos naturais de vida.

Fundamentais para a construção de tal enquadramento são (i) o reconhecimento da natureza interactiva da funcionalidade e do desenvolvimento humano, preconizado pelo modelo bioecológico de Bronfrenbrenner; (ii) as 
teorias Transaccionais que destacam a natureza dinâmica do desenvolvimento e realçam a influência mútua da pessoa e do seu ambiente. A natureza e a extensão da realização de actividades e da participação social definem-se, mutuamente, pelas características da pessoa e pelas características do ambiente; (iii) o reconhecimento da incapacidade como sendo um fenómeno multidimensional, o que requer uma avaliação diferenciada em termos dos componentes da interacção pessoa/ambiente (Pinto, Grande, Novais, Rosário, \& Barbieri, 2009; Simeonsson et al., 2003).

Tendo como enquadramento conceptual as abordagens acima referidas, a Organização Mundial de Saúde (OMS) equacionou um modelo das interacções pessoa-meio (ver Figura 1) como base para a elaboração de uma classificação rigorosa da funcionalidade humana.

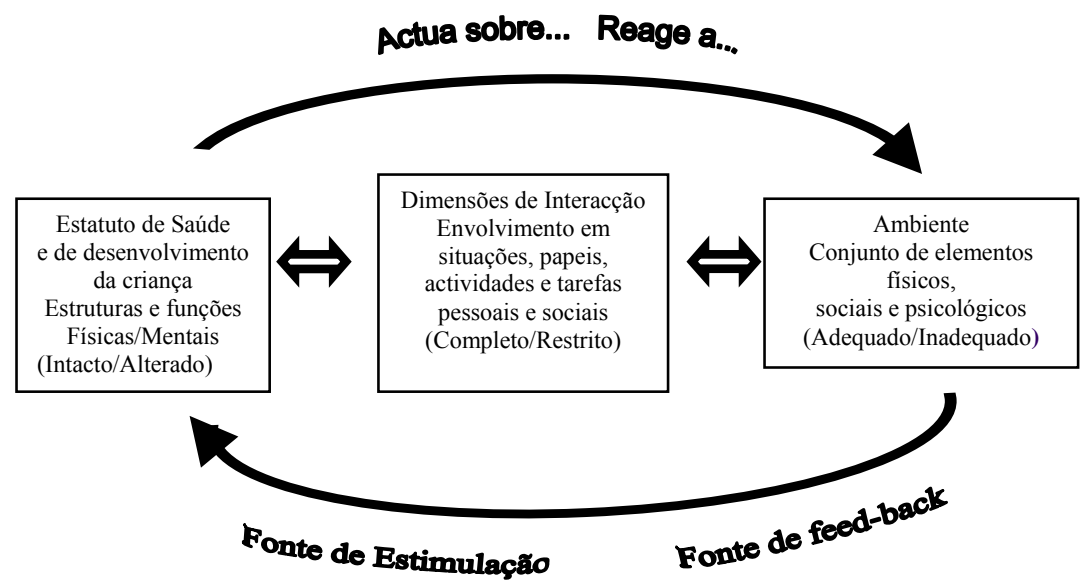

Figura. 1 Modelo da relação entre variáveis - Modelo Biopsicossocial (OMS, 2003)

Esta abordagem vem ao encontro das preocupações expressas por Bairrão (1994), ao realçar a necessidade de um enquadramento que englobe as concepções acerca da natureza ecológica da funcionalidade da criança, da sua saúde e desenvolvimento. Desta forma, a avaliação e a classificação da funcionalidade deve reconhecer a natureza idiossincrática e complexa da criança com incapacidades, o que requer o recurso a abordagens abrangentes que vão além dos métodos psicométricos, nosológicos e nosográficos (Simeonsson \& Rosenthal, 2001). 


\section{Intervenção Precoce (IP) e Educação Especial (EE) - Questões de Avaliação, de Diagnóstico e de Classificação}

Os avanços da ciência no campo do desenvolvimento humano têm vindo a reforçar a noção de que a atribuição de um diagnóstico pode revelar pouco, ou muito pouco, daquilo que são as características de funcionalidade da criança. Os diagnósticos estão relacionados com sintomatologias e poucas vezes aparecem relacionados com a função e, mais ainda, não a predizem. É frequente que as características de crianças com o mesmo diagnóstico difiram mais do que aquelas de crianças com diagnósticos diferentes (Lollar \& Simeonsson, 2005). Embora os diagnósticos sejam importantes para definir causas e prognósticos, no caso de crianças com incapacidades há uma grande variabilidade na forma como realizam as actividades da vida diária, bem como na forma como participam na sociedade, pelo que as categorias diagnósticas não devem ser utilizadas como informação privilegiada na planificação e implementação das intervenções. Simultaneamente, os contextos onde as crianças vivem (físicos, sociais e psicológicos) influenciam a sua funcionalidade, sendo o reconhecimento da importância dessa influência fundamental para se procurarem as melhores formas de intervenção. Na área da Educação, esta situação tem especial relevo, uma vez que a identificação dos alunos por categorias é, muitas vezes, uma barreira à intervenção (Simeonsson et al., 2003).

Assim, a existência de uma classificação com base num modelo conceptual ecológico-sistémico, de carácter universal e devidamente adaptada a crianças e jovens, surge como um grande contributo para a caracterização das crianças, tendo como base um perfil funcional e não um rótulo diagnóstico.

O desenvolvimento da CIF-CJ (WHO, 2007) reflecte, então, uma mudança conceptual da classificação das consequências da doença para uma classificação dos componentes da saúde, providenciando, igualmente, uma abordagem abrangente e unificadora da incapacidade e da funcionalidade humana. $O$ objectivo geral da classificação é proporcionar uma linguagem unificada e padronizada e uma estrutura que descreva a saúde e os estados relacionados com a saúde. Define os componentes da saúde e alguns componentes do bem-estar relacionados com a saúde (tais como a educação e o trabalho). Os domínios contidos na CIF podem, portanto, ser considerados como domínios da saúde [ver, ouvir, aprender, lembrar, etc.] e domínios relacionados com a saúde [transporte, educação, interaç̧ões sociais, etc.] (OMS, 2003, pp. 5), o que é consistente com a definição de saúde como um estado dinâmico de completo bem-estar físico, mental e social e não apenas a ausência de doença ou enfermidade (WHO, 1948). 
Assim, a funcionalidade é descrita (utilizando-se um sistema de codificação alfanumérica) numa interacção dinâmica entre três dimensões ou componentes: (i) funções/estruturas do corpo, que abrangem os sistemas fisiológicos ou anatómicos; (ii) actividade e participação, que descrevem as áreas de vida; (iii) factores ambientais, que englobam o ambientem físico, social e atitudinal. Os factores pessoais são tomados em consideração na descrição da funcionalidade, mas não são alvo de codificação (ver Figura 2).

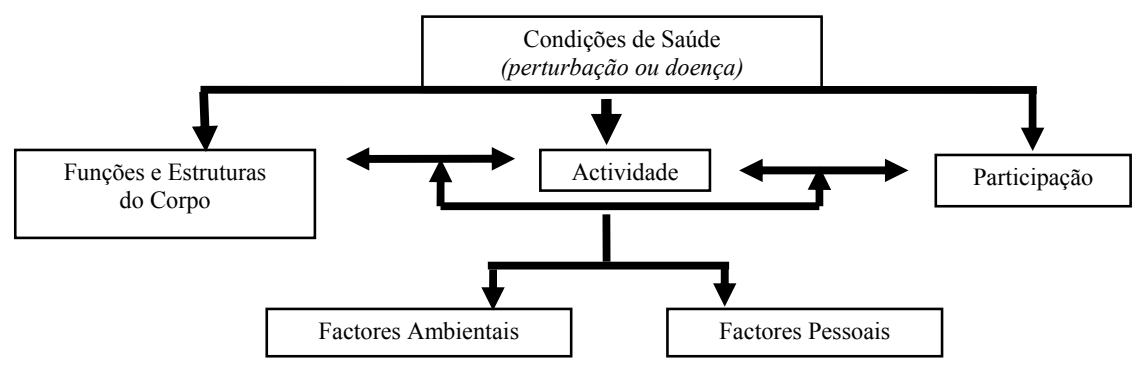

Figura 2. Modelo da CIF - Organização Mundial de Saúde (2003)

A CIF-CJ não é um instrumento de avaliação, mas antes uma ponte para a interpretação e sumarização dos resultados dos instrumentos de avaliação da funcionalidade. A utilização dos códigos da CIF-CJ auxilia na organização da informação, clarifica a avaliação realizada e facilita a comunicação entre técnicos, família e serviços. Assim, as unidades de classificação são as características funcionais e não os diagnósticos clínicos. Os perfis funcionais resultantes da classificação são, em primeiro lugar e mais do que tudo, descritivos dos factores que definem as dificuldades da criança em cumprir as exigências do dia-a-dia. A base funcional destes perfis, particularmente na realização das actividades, deve ter implicações práticas e directas no desenvolvimento de intervenções individualizadas e de planos de tratamento. Neste sentido, a documentação da interacção criança-ambiente é útil na identificação das barreiras e dos facilitadores que podem mediar as aprendizagens e a adaptação da criança. Deste modo, podem ser seleccionadas as variáveis adequadas para planear a intervenção e documentar os seus resultados, bem como os resultados desenvolvimentais das crianças (essencialmente, da área de actividade e participação), permitindo uma maior ligação entre a identificação das limitações e a natureza da intervenção (Lollar \& Simeonsson, 2005).

A CIF-CJ constitui-se como um procedimento unificador para o trabalho interdisciplinar através da utilização dos códigos, enquanto linguagem universal para definir as características de funcionalidade e saúde. Assim, os 
diagnósticos, por si só, bem como as alterações biomédicas (das estruturas e funções do corpo) não permitem responder às necessidades de apoio das crianças. A natureza do processo de avaliação-intervenção deverá ser largamente determinada pelo estatuto funcional da criança, pelas actividades que é ou não capaz de realizar, pelo seu nível de participação e pelos factores ambientais envolvidos neste processo (Figura 3).

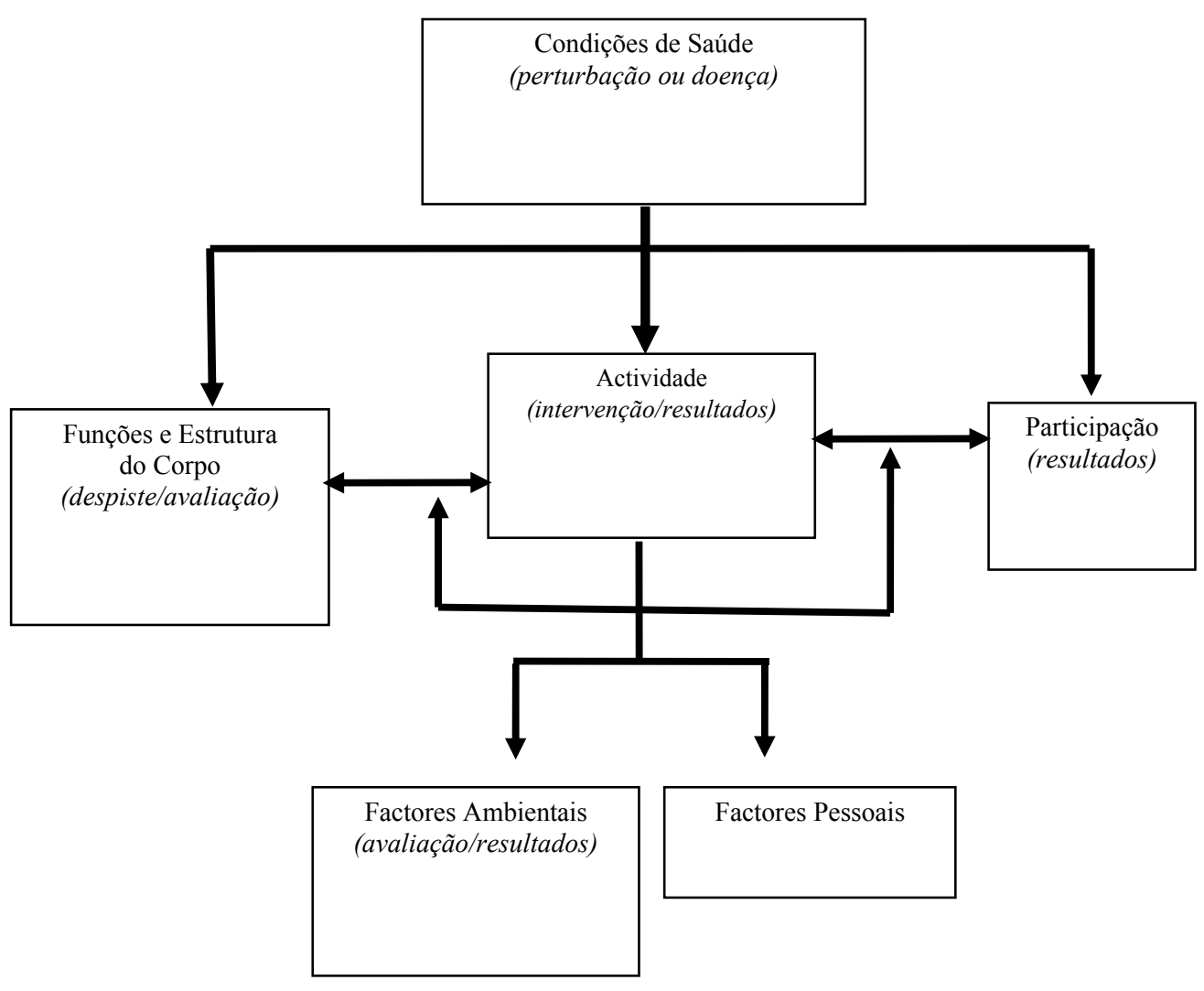

Figura 3. Modelo da CIF-CJ no processo de avaliação/intervenção (adaptado de Lollar e Simeonsson, 2003) 


\section{Questões de correspondência entre instrumentos de avaliação e os domínios da CIF e CIF-CJ}

Uma das prioridades que surge com a utilização da classificação diz respeito à análise de medidas de avaliação que sejam compatíveis com o seu conteúdo (Simeonsson et al., 2003).

Vários estudos têm demonstrado que, em geral, a CIF e a CIF-CJ são instrumentos úteis na descrição da funcionalidade de crianças, em diferentes áreas de avaliação/intervenção e com diferentes tipos de incapacidades. Simultaneamente, demonstram que há vários instrumentos de avaliação que são compatíveis com o conteúdo da CIF e da CIF -CJ (Battaglia, Russo, Bolla, Chiusso, Bertelli, Pellegr, Borri, G \& Martinuzzi, 2004; Law, Finkelman, Hurley, Rosenbaum, King, King, \& Hanna, 2004; Grandlund, Eriksson, \& Ylvén, 2004; Pernboom, \& Chorus, 2003; Simeonsson. et al., 2003)

De seguida, apresentamos, a título exemplificativo, alguns dos inúmeros instrumentos para os quais foram realizados estudos de correspondência entre os seus itens e os domínios da Classificação (Quadro 1). Em Portugal estão agora a iniciar-se estudos de correspondência de instrumentos utilizados na área da IP, nomeadamente por uma equipa da Faculdade de Psicologia e de Ciências da Educação da Universidade do Porto (FPCE UP)

\section{Linhas de investigação na FPCE UP}

Na FPCE UP têm vindo a ser desenvolvidas investigações com o objectivo de analisar a utilidade e aplicabilidade da CIF-CJ no processo de avaliação-intervenção em IP e EE. As linhas orientadoras das investigações, todas elas ainda em curso, vão no sentido de: (i) descrever os perfis funcionais das crianças em serviços de Intervenção Precoce e crianças em contexto de creche; (ii) desenvolver um instrumento tendo por base o referencial da CIF CJ, contendo indicadores úteis no despiste de crianças e na elegibilidade para serviços de Intervenção Precoce; (iii) criar uma base de dados de instrumentos de avaliação de crianças com Perturbações do Espectro do Autismo compatíveis com a CIF-CJ; (iv) verificar a utilidade de uma metodologia de avaliação-intervenção da Participação das crianças com Perturbações do Espectro do Autismo, em contexto escolar, que utiliza a CIF-CJ como suporte técnico e teórico; (v) analisar as características dominantes dos alunos sinalizados para os serviços de Educação Especial, bem como daqueles cuja avaliação especializada indicou como não elegíveis, antes e após a introdução do Decreto-Lei 3/2008; (vi) averiguar de que forma o uso da classifica- 
ção se repercutiu sobre o focus de análise dos profissionais na documentação da incapacidade e na definição de objectivos e métodos de intervenção.

Quadro 1. Exemplos de correspondência estabelecida entre instrumentos de avaliação e os domínios da CIF e CIF-CJ

\begin{tabular}{|c|c|c|}
\hline $\begin{array}{l}\text { The Pediatric Evaluation of } \\
\text { Disability Inventory (PEDI) } \\
\text { Haley, Coster, Ludlow, Halti- } \\
\text { wanger, \& Andrellos (1992) } \\
\text { Dos } 8 \text { meses aos } 6 \text { anos de } \\
\text { idade }\end{array}$ & $\begin{array}{l}\text { Avaliação clínica de } \\
\text { capacidades funcionais, } \\
\text { da realização de activida- } \\
\text { des e das mudanças nas } \\
\text { competências funcionais }\end{array}$ & $\begin{array}{l}\text { Actividade e Parti- } \\
\text { cipação } \\
\begin{array}{l}\text { Factores Ambien- } \\
\text { tais }\end{array}\end{array}$ \\
\hline $\begin{array}{l}\text { Educational Needs Question- } \\
\text { naire } \\
\text { Victoria Department of Educa- } \\
\text { tion, Australia (s/d) }\end{array}$ & $\begin{array}{l}\text { Descrição das competên- } \\
\text { cias da criança em } 12 \\
\text { domínios diferentes }\end{array}$ & $\begin{array}{l}\text { Actividade e Parti- } \\
\text { cipação } \\
\text { Factores Ambien- } \\
\text { tais }\end{array}$ \\
\hline $\begin{array}{l}\text { Vineland Adaptive Scales } \\
\text { Sparrow, Balla, \& Cicchetti } \\
\text { (1984) } \\
\text { Desde o nascimento até à idade } \\
\text { adulta }\end{array}$ & $\begin{array}{l}\text { Avaliação de competên- } \\
\text { cias pessoais e sociais }\end{array}$ & $\begin{array}{l}\text { Actividade e Parti- } \\
\text { cipação }\end{array}$ \\
\hline $\begin{array}{l}\text { London Handicap Scale } \\
\text { Harwood, Rogers, Dickinson, \& } \\
\text { Ebrahim (1994) }\end{array}$ & $\begin{array}{l}\text { Determinação do efeito de } \\
\text { doenças crónicas nas } \\
\text { competências funcionais }\end{array}$ & $\begin{array}{l}\text { Funções do Corpo } \\
\text { Actividade e Parti- } \\
\text { cipação }\end{array}$ \\
\hline $\begin{array}{l}\text { Perceived Interaction } \\
\text { Granlund, \& Bjorck-Akesson } \\
\text { (1999) }\end{array}$ & $\begin{array}{l}\text { Auto-avaliação do envol- } \\
\text { vimento em actividades } \\
\text { da vida diária }\end{array}$ & Participação \\
\hline $\begin{array}{l}\text { The Abilities Index } \\
\text { (Simeonsson \& Bailey, 1984) }\end{array}$ & $\begin{array}{l}\text { Descrição do perfil fun- } \\
\text { cional das competências e } \\
\text { limitações da criança }\end{array}$ & $\begin{array}{l}\text { Estruturas e Fun- } \\
\text { ções do Corpo } \\
\text { Actividade e Parti- } \\
\text { cipação }\end{array}$ \\
\hline $\begin{array}{l}\text { Functional Independence } \\
\text { Measure }\left(\mathrm{FIM}^{\mathrm{TM}}\right) \\
\text { Wright }(2000)\end{array}$ & $\begin{array}{l}\text { Avaliação da independên- } \\
\text { cia funcional }\end{array}$ & $\begin{array}{l}\text { Funções do Corpo } \\
\text { Actividade e Parti- } \\
\text { cipação }\end{array}$ \\
\hline
\end{tabular}




\section{Conclusão}

A CIF-CJ apresenta-se como uma ferramenta útil, na medida em que classifica as várias dimensões do funcionamento humano. É uma alternativa às classificações baseadas em questões etiológicas ou de diagnóstico e apresenta uma taxonomia e linguagem universais passíveis de incrementar as trocas interdisciplinares de informação ao nível das políticas, da investigação e da prática a nível mundial (Unstun, Chatterji, Bickenbach, Kostanjesk, \& Schneider, 2003). Apesar da progressiva e crescente utilização da classificação, a sua implementação implica mudanças importantes a nível conceptual que se reflictam directamente nas práticas (nomeadamente no que respeita às questões de avaliação e à necessidade crescente de um trabalho de equipa interdisciplinar), nas políticas e nas entidades prestadoras de serviços. Simultaneamente, é importante que a investigação explore aspectos que sirvam para uma melhor operacionalização da classificação e sua consequente utilização, nomeadamente no que respeita: (i) ao desenvolvimento de instrumentos de avaliação consentâneos com o referencial teórico da CIF-CJ; (ii) ao estudo de instrumentos compatíveis com esse referencial; (iii) à utilização da CIF-CJ para a definição de critérios de elegibilidade, para a implementação e avaliação da eficácia de serviços (Ustun et al., 2003).

A CIF-CJ surge como um contributo valioso para o desenvolvimento de uma perspectiva abrangente do desenvolvimento e da funcionalidade das crianças com incapacidades, constituindo provavelmente o único sistema de classificação sistemática conciliando a saúde, a aprendizagem e o funcionamento social num sistema coerente e operacional (McAnaney, 2007, citado em Felgueiras, 2009, pp. 206).

\section{Referências}

Bairrão, J. (1994). A perspectiva ecológica na avaliação de crianças com necessidades educativas especiais e suas famílias: $\mathrm{O}$ caso de intervenção precoce. Inovação, 7, 37-48.

Bairrão, J. (2003). Understanding functioning and disability in early childhood: Iintervention theories and models. Comunicação apresentada no EU-FIPSE-International Programme in Early childhood Intervention. Suécia: Mälardalen Högskola, Västeros.

Battaglia, M., Russo, E., Bolla, A., Chiusso, A., Bertelli, S., Pellegr, A., Borri, G., \& Martinuzzi, A. (2004). International Classification of Functioning, Disability and Health in a cohort of children with cognitive, motor and complex disabilities. Developmental Medicine \& Child Neurology, 46, 98-106.

Felgueiras, I. (2009). A Classificação Internacional da Funcionalidade, Incapacidade e Saúde (CIF) e os seus contributos para a reconceptualização da deficiência e 
incapacidade. In G. Portugal, Ideias, projectos e inovação no mundo das infâncias: O percurso e a presença de Joaquim Bairrão. (pp. 195-209). Aveiro: Theoria Poiesis Praxis, Universidade de Aveiro.

Law, M., Finkelman, S., Hurley, P., Rosenbaum, P., King, S., King, G., \& Hanna, S. (2004). Participation of children with physical disabilities: Relationships with diagnosis, physical function, and demographic variables. Scandinavian Journal of Occupational Therapy, 11, 156-162.

Lollar, D., \& Simeonsson, R. J. (2005). Diagnosis to function: Classification for children and youths. Developmental and Behavioral Pediatrics, 26 (4), $323-$ -330 .

Grandlund, M., Eriksson, L., \& Ylvén, R. (2004). Utility of International Classification of Functioning, Disability and Health's Participation dimension in assigning ICF codes to items from extant rating instruments. Journal of Rehabilitation Medicine, 36, 130-137.

Ogonowski, J., Kronk, R., Rice, C., \& Feldman, H. (2004). Inter-rater reliability in assigning ICF codes to children with disabilities. Disability and Rehabilitation, $26(6), 353-361$.

Organização Mundial de Saúde (2003). Classificação Internacional da Funcionalidade, Incapacidade e Saúde. [Transl. from International Classification of Functioning, Disability and Health (WHO, 2001)]. Lisboa: Secretariado Nacional de Reabilitação.

Pernboom, R. J., \& Chorus, A. M. J. (2003). Measuring participation according to the International Classification of Functioning (ICF), Disability and Rehabilitation, 25 (11-12), 557-587.

Pinto, A. I., Grande, C., Novais, I., Rosário, H., \& Barbieri, M. (2009). Intervenção precoce: Uma abordagem dimensional do desenvolvimento humano. In G. Portugal, Ideias, projectos e inovação no mundo das infâncias: $O$ percurso e a presença de Joaquim Bairrão (pp.63-76). Aveiro: Theoria Poiesis Praxis, Universidade de Aveiro.

Pinto, A. I., Pessanha, M., Barros, S., Grande, C., Aguiar, C., \& Nunes, C. (2009). Educação e investigação em idades precoces. In G. Portugal, Ideias, projectos e inovação no mundo das infâncias: O percurso e a presença de Joaquim Bairrão. (pp. 47-62). Aveiro: Theoria Poiesis Praxis, Universidade de Aveiro.

Simeonsson, R. J. (2003). Classification of communication disabilities in children: Contribution of the International Classification of Disability and Health. International Journal of Audiology, 42, S2-S8.

Simeonsson, R. J., Leonardi, Lollar, D. J., M., Bjork-Akesson, E., Hollenweger, J., Brown, S. C., \& Martinuzzi, A. (2003). Applying the International Classification of Functioning, Disability and Health (ICF) to measure child disability. Disability and Rehabilitation, 25 (11-12), 602-610.

Simeonsson, R., Pereira, S., \& Scarborough, A. (2003), Documenting delay and disability in early development with the WHO-ICF. Psicologia, XVII, 31-41.

Simeonsson, R. J., \& Rosenthal, S. L. (2001). Clinical assessment of children: An overview. In R. J. Simeonsson \& S. L. Rosenthal (Eds.), Psychological and developmental assessment. Children with disabilities and chronic conditions. New York: The Guilford Press. 
Unstun, T. B., Chatterji, S., Bickenbach, J., Kostanjesk, N., \& Schneider, M. (2003). The International Classification of Functioning Disability and Health: A new tool for understanding disability and health. Disability and Rehabilitation, 25 (11-12), 565-571.

World Health Organization (1948). Preamble to the Constitution of the World Health Organization as adopted by the International Health Conference, New York, 19-22 June, 1946; signed on 22 July 1946 by the representatives of 61 States (Official Records of the World Health Organization, n. ${ }^{\circ} 2$, p. 100) and was enforced on April 7, 1948.

World Health Organization, (2001). International Classification of Functioning, Disability and Health. Geneva: World Health Organization.

World Health Organization, (2007). International Classification of Functioning, Disability and Health - Children and Youth. Geneva: World Health Organization. 